\title{
ANÁLISIS DEL CICLO PHVA EN LA GESTIÓN DE PROYECTOS, UNA REVISIÓN DOCUMENTAL
}

\author{
Faber Andrés Moyano Hernández ${ }^{1}$, Diana Carolina Villamil Sandoval ${ }^{2}$.
}

${ }^{1}$ Ingeniero Industrial, Estudiante de Maestría en Gerencia de Proyectos, Universidad Militar Nueva Granada, Kilómetro 2, vía Cajicá - Zipaquirá (Colombia). u20800283@unimilitar.edu.co

${ }^{2}$ Magister en ingeniería con especialidad en Sistemas de Calidad y Productividad, Docente de la Universidad Militar Nueva Granada, Kilómetro 2, vía Cajicá - Zipaquirá (Colombia). diana.villamil@unimilitar.edu.co

\section{RESUMEN}

El ciclo PHVA ha sido utilizado ampliamente para la mejora de los procesos; por ello, es adecuado pensar que es una herramienta útil para la gestión de proyectos. El objeto de la presente investigación es evidenciar los principales aportes que han realizado diferentes autores en la implementación del ciclo PHVA para la mejora continua en la gestión de proyectos. Se realizó una revisión documental, empleando estrategias de búsqueda con palabras clave en Scopus y ScienceDirect. Se recopilaron documentos de los últimos 5 años, resultando un total de 186 artículos. Al verificar la pertinencia con el tema de estudio, la lista se redujo a 21 . Se destacan como resultados la vigencia temática del ciclo PHVA, la variabilidad de sectores empresariales y los países que la implementan y estudian. El continente europeo lidera el volumen de publicaciones y a pesar de encontrar alto volumen de artículos, muchos se descartaron por debilidades metodológicas.

Palabras clave: Ciclo PHVA; gestión de proyectos; mejoramiento continuo; productividad; fases de la administración de proyectos.

Recibido: 26 de mayo de 2021. Aceptado: 20 de julio de 2021

Received: May 26, 2021. Accepted: July 20, 2021

DOI: https://doi.org/10.33571/rpolitec.v17n34a4

\section{ANALYSIS OF THE PDCA CYCLE IN PROJECT MANAGEMENT, A DOCUMENTARY REVIEW}

\begin{abstract}
The PDCA cycle has been widely used for process improvement; therefore, it is appropriate to think that it is a useful tool for project management. The purpose of this research is to show the main contributions that different authors have made in the implementation of the PDCA cycle for continuous improvement in project management. A documentary review was carried out, using search strategies with keywords in Scopus and Science Direct. Documents from the last 5 years were collected, resulting in a total of 186 articles. When verifying the relevance to the subject of study, the list was reduced to 21 . The thematic validity of the PDCA cycle, the variability of business sectors and the countries that implement and study it stand out as results. The European continent leads the volume of publications and despite finding a high volume of articles, many were discarded due to methodological weaknesses.
\end{abstract}

Keywords: PDCA cycle; Projects management; continuous improvement; productivity; phases of project management.

Cómo citar este artículo: F.A. Moyano y D.C. Villamil. "Análisis del Ciclo PHVA en la Gestión de Proyectos", Revista Politécnica, vol.17, no.34 pp.55-69, 2021. DOI: https://doi.org/10.33571/rpolitec.v17n34a4 


\section{INTRODUCCIÓN}

El análisis del uso del ciclo PHVA en la gestión de proyectos, nace a partir de los beneficios que genera esta herramienta de mejora continua, sobre los procesos de las organizaciones que la aplican; las cuales logran percibir mejoras en un corto plazo con resultados visibles; tales como la reducción de productos defectuosos, la disminución en costos y el menor tiempo, aspectos que representan a las variables de la triple restricción que debe sortear cualquier tipo de proyecto. Además la herramienta genera el incremento de la productividad, promoviendo la competitividad en el sector propio de la organización.

Por este motivo la integración de esta herramienta, en la gestión de proyectos busca orientar la calidad en los procesos y la toma de decisiones para la gestión de los recursos, el cronograma y los costos, en el desarrollo de diferentes tipos de proyectos. Este artículo muestra entonces el enfoque de la literatura expuesta por diferentes autores que han investigado sobre el tema, de forma que se pueda fortalecer dentro de las organizaciones, a las áreas encargadas de la gestión proyectos, con algunos ejemplos de incorporación de esta herramienta en los procesos de gestión anteriormente enunciados.

El rápido crecimiento del conocimiento científico ha provocado un volumen de publicaciones electrónicas en internet, que hace imposible a cualquier profesional o tomador de decisiones, mantenerse al tanto de la nueva evidencia, razón por la cual se han desarrollado investigaciones de re-visión bibliográfica o documental que permiten sintetizar altos volúmenes de evidencia científica, al servicio de la comunidad académica [1], razón por la cual, la presente investigación desarrolló una búsqueda estructurada metodológicamente sobre un tema trascendental como la implementación del ciclo PHVA en la mejora continua de gestión de proyectos.

Teniendo en cuenta la relevancia de ciclo PHVA a nivel empresarial, la presente revisión documental se realizó con el objeto de sintetizar la mejor evidencia científica relacionada con la implementación del ciclo PHVA en la mejora continua de la gestión de proyectos.

\section{MATERIALES Y MÉTODO}

Este artículo corresponde a un proceso exploratorio desde el cual se pretende desarrollar un análisis cuantitativo, de la mejor bibliografía disponible en relación con la implementación del ciclo PHVA a nivel empresarial para la mejora continua de la gestión de proyectos, esta metodología de análisis es ampliamente utilizada por autores top, de artículos originales como Barringer, et al [2].

La población objeto de estudio corresponde a la literatura publicada en los últimos 5 años sobre el impacto de la implementación del ciclo PHVA en la mejora continua de la gestión de proyectos, en la base de datos bibliográfica Scopus, considerada una de las mayores bases de datos de citas y resúmenes de literatura revisada por pares, incluyendo revistas científicas, libros y actas de conferencias [3] y en ScienceDirect que relacionada hasta 1.4 millones de artículos de acceso libre; recogiendo entre ambas un alto porcentaje de la literatura científica del mundo.

La búsqueda fue realizada utilizando diferentes tipos de Query, por medio de los módulos de búsquedas avanzadas en cada una de las bases de datos, estos Query se construyeron en inglés y utilizando los conectores booleanos de OR para realizar las búsquedas iniciales, que permitieron mayor amplitud, el conector AND para las búsquedas posteriores que permitieron mayor especificidad y el NOT para excluir artículos que estaban aparentemente relacionados pero que al revisar correspondían a falsos positivos como por ejemplo el "benchmarking".

La estrategia de búsqueda utilizada fue:

a. Cycle PDCA OR Projects management OR

b. Cycle PDCA AND Projects management AND

c. Cycle PDCA AND Projects management NOT Benchmarking 
Cada una de estas tres estrategias de búsqueda fue realizada en Scopus y ScienceDirect, empleando los algoritmos de búsqueda alcanzados que generan los Query necesarios para lograr la búsqueda, reportando los hallazgos relacionados en la Tabla 1 que corresponden a 186 documentos.

Tabla 1. Resultados de la estrategia de búsqueda en Scopus y ScienceDirect 2021.

\begin{tabular}{|c|c|c|c|}
\hline \multirow{2}{*}{$\begin{array}{c}\text { Base de datos } \\
\text { bibliográfica }\end{array}$} & \multicolumn{2}{|c|}{ Variables } & Interrelación de Variables \\
\cline { 2 - 4 } & Cycle PDCA & $\begin{array}{c}\text { Project } \\
\text { management }\end{array}$ & $\begin{array}{c}\text { Project management and PDCA } \\
\text { cycle NOT Benchmarking }\end{array}$ \\
\hline $\begin{array}{c}\text { Scopus (filters: engineering/ } \\
\text { 2016-2021) }\end{array}$ & 22 & 70 & 20 \\
\hline $\begin{array}{c}\text { ScienceDirect (filters: engineering/ } \\
\text { 2016-2021) }\end{array}$ & 50 & 21 & 23 \\
\hline $\begin{array}{c}\text { Total de } \\
\text { Artículos }\end{array}$ & 72 & 91 & 2 \\
\hline
\end{tabular}

Con los 186 artículos encontrados se realizó un proceso de depuración de duplicados, utilizando del gestor de referencias Zotero y validándolo posteriormente en Microsoft Excel $®$, encontrando un total de 27 artículos que se repitieron en las dos bases de datos, los cuales fueron eliminados, quedando un total de 157 artículos preseleccionados.

Con los artículos preseleccionados se realizó un proceso de depuración a partir de la lectura del resumen y la introducción de cada publicación, escogiendo únicamente los artículos en los que el autor trata temáticas a fines con la calidad en los procesos para la variable del ciclo PHVA y en temas de gestión de alcance, recursos, cronograma y costos, para la variable de gestión de proyectos, a fin de poder orientar a través de las interpretaciones y conclusiones de cada autor, a la revisión documental de este artículo, teniendo en cuenta además los siguientes criterios: que fueran artículos originales, con fecha de publicación entre 2016 y 2021 , que implementaran el ciclo PHVA y que evaluaran la mejora continua en la administración de proyectos. Una vez superados estos criterios se excluyeron un total de 136 artículos, quedando así 21 papers finales con los cuales se realizó el análisis.

En ese sentido, para el desarrollo de este trabajo, se seleccionaron y analizaron un total de 21 publicaciones de un total de 186 que abordaron el tema de Ciclo PHVA y gestión de proyectos, como temática individual, o relacionadas entre sí, resaltando que no se encontró un gran número de estudios que vinculen estas variables entre sí. La Tabla 2, muestra los resultados en número de publicaciones obtenidos por cada una de las consultas realizadas a cada variable de investigación, en las bases de datos bibliográficas, enunciadas anteriormente. Cabe resaltar que el ítem de selección de cada documento, muestra el número de publicaciones seleccionadas en cada una de las consultas realizadas a cada variable de investigación, en las bases de datos bibliográficas, enunciadas anteriormente.

Tabla 2. Selección de artículos, resultado de la búsqueda en bases de datos bibliográfica por variables de investigación.

\begin{tabular}{|c|c|c|c|}
\hline \multirow{2}{*}{$\begin{array}{c}\text { Base de datos } \\
\text { bibliográfica }\end{array}$} & Cycle PDCA & $\begin{array}{c}\text { Project manage- } \\
\text { ment }\end{array}$ & $\begin{array}{c}\text { Project management and PDCA } \\
\text { cycle NOT Benchmarking }\end{array}$ \\
\cline { 2 - 4 } & 4 & 2 & 5 \\
\hline Scopus & 3 & 3 & 4 \\
\hline ScienceDirect & 7 & 5 & 9 \\
\hline Total de Artículos & & & \\
\hline
\end{tabular}


Con los artículos seleccionados se realizó un análisis descriptivo, por medio de frecuencias absolutas y relativas, presentadas en tablas de resumen, de los resultados obtenidos donde se evaluó el año de publicación, el lugar, la revista de publicación, el sector de empresa, el tipo de ciclo PHVA, el tipo de proyecto implementado, los objetivos del proyecto donde se construyó una variable con tres categorías, que corresponde a los puntos comunes de los artículos que fueron: la gestión de proyectos, la gestión de calidad y la gestión de costos y los resultados o conclusión obtenida por el paper.

\section{RESULTADOS}

Para lograr identificar los 21 estudios de la presente revisión documental, se excluyeron un total de 163 artículos, de los cuales el $16,6 \%$ se excluyó por duplicidad, entre los resultados de la búsqueda por estrategia y base de datos, mientras que el $83,4 \%$ de artículos restantes fueron excluidos de acuerdo a los criterios de tipificación establecidos, donde hubo la necesidad de leer el resumen y en algunos casos el apartado metodológico que se observa en la Fig. 1.

Lo anterior evidencia que el proceso de selección de los artículos no fue realizado aleatoriamente ni por conveniencia del investigador y si bien esta revisión, no corresponde a una revisión sistemática representativa de toda la evidencia temática actual, si es una revisión bibliográfica reproducible, dada la claridad metodológica, estructurada y organizada con la que se llegó a los 21 artículos finales y que se evidencia en la Fig. 1 y la Tabla 3.

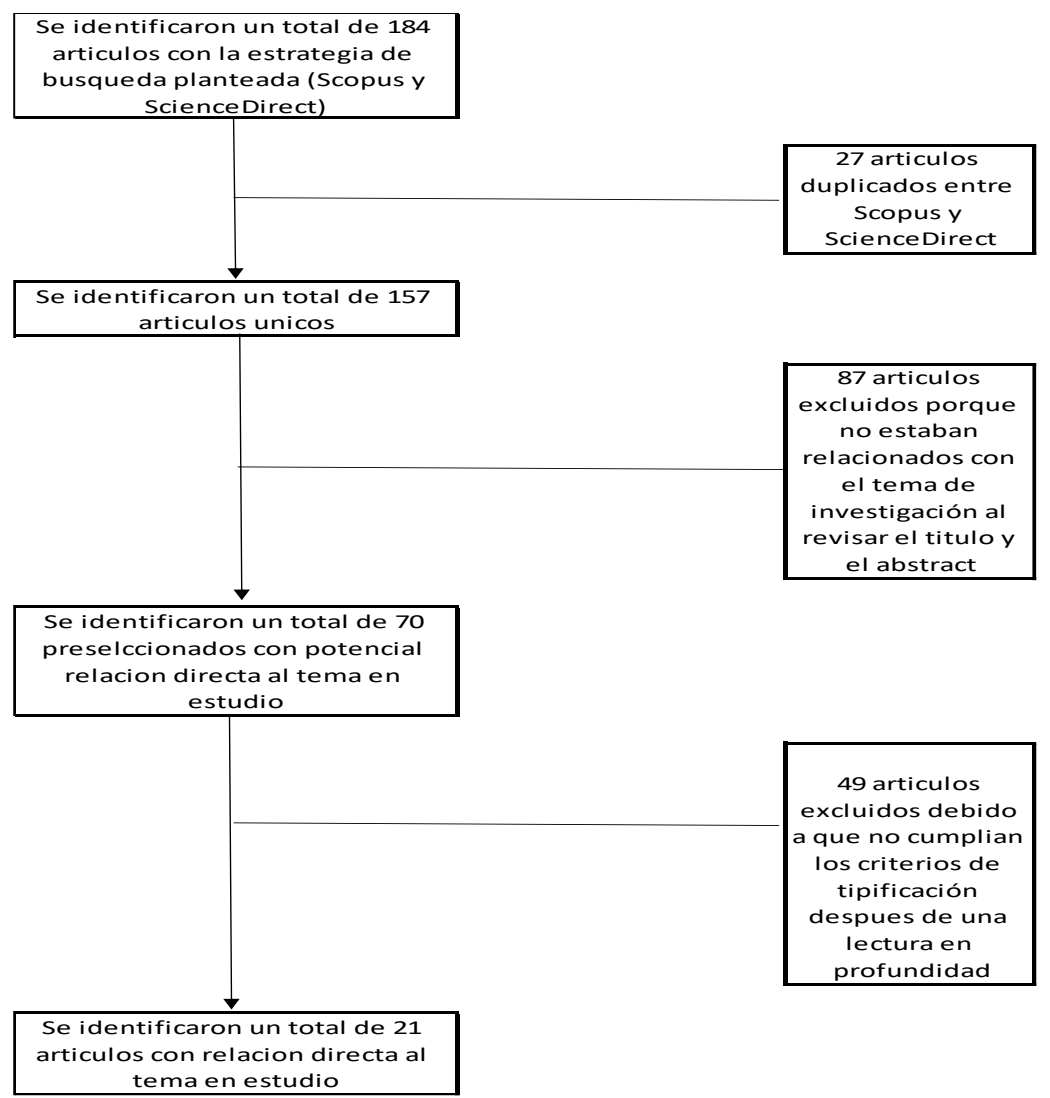

Fig. 1. Flujograma del proceso de selección de artículos de la revisión bibliográfica.

Si bien las estrategias de búsqueda fueron las mismas en ambas bases de datos, se encontró que el $52,4 \%$ de los artículos provino de Scopus, los 21 artículos se publicaron en un total de 18 revistas indexadas, donde 
solo repitieron artículos tres revistas, con dos publicaciones cada una, siendo estas: Journal of Cleaner Production de Elsevier, revista internacional y multidisciplinaria centrada en la investigación y la práctica de Producción Limpia y Sostenible con el Medio Ambiente, cuyo propósito general es ayudar a las sociedades a ser más sostenibles [4]; la segunda revista fue Procedia CIRP de Elsevier, producto de acceso abierto centrado en la publicación de actas de alta calidad de conferencias CIRP (Academia Internacional para la Producción de Ingeniería) [5] y por ultimo Procedia Engineering de Elsevier, revista sobre procesos e ingeniería, que fue descontinuada desde 2021. En la Tabla 3 se muestran las características demográficas temporales y espaciales de los 21 artículos seleccionados en la revisión bibliográfica.

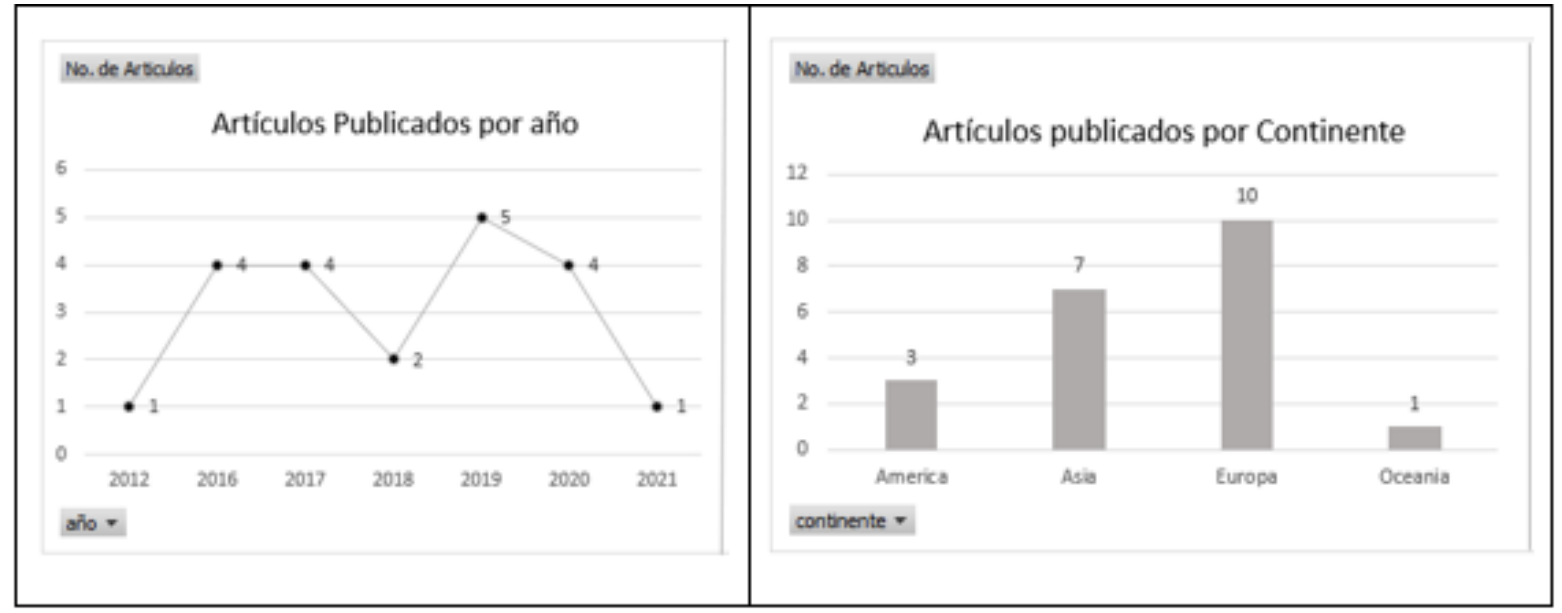

Fig. 2. Comportamiento temporal y espacial de las 21 publicaciones seleccionadas.

En la Gráfica 2 se observa que los años con menor frecuencia de artículos que han abordado la mejora continua en las fases de la gestión de proyectos, mediante la aplicación del ciclo PHVA, fueron 2012 con un solo artículo y 2018 con 2 artículos, mientras el año con mayor volumen de publicaciones en la temática abordada, fue el 2019 con 5 artículos; el comportamiento temporal no permite identificar ningún tipo de patrón positivo o negativo en las frecuencias de publicación del tema en estudio, sin embargo al excluir el 2012 que fue un artículo incluido debido a la relevancia metodológica que aportaba, a pesar que no cumplía el criterio temporal y excluir el 2021 puesto que solo participó con un trimestre, se podría evidenciar consistencia en el número de publicaciones entre 4 y 5 exceptuando únicamente el año 2018.

En cuanto a la distribución espacial de las publicaciones en el Gráfico 2 se muestra un claro ganador, siendo este el Continente Europeo con 10 de las 21 publicaciones equivalentes al 47,6\%, seguido por los países Asiáticos con el 33,3\% y en tercer lugar el Continente Americano con el 14,3\%; solo fue seleccionado un artículo realizado por autores latinoamericanos, específicamente en Perú, realizado por Buitron-Lopez, L., et al, presentado en la CONCAPAN en 2019 en ciudad de Panamá.

Los sectores empresariales con mayor volumen de referencias bibliográficas fueron principalmente tres: el industrial con el $38,1 \%$, seguido por la Construcción con el $28,6 \%$ y el energético con $14,3 \%$, sumando entre los tres el $81 \%$ del total de artículos seleccionados en la presente revisión sobre abordado la mejora continua en las fases de la gestión de proyectos, mediante la aplicación del ciclo PHVA como se observa en la Tabla 3.

Tabla 3. Características demográficas temporales y espaciales de los 21 artículos seleccionados en la revisión bibliográfica. 


\begin{tabular}{|c|c|c|c|c|c|c|c|c|}
\hline No & $\begin{array}{l}\text { BASE } \\
\text { DE DA- } \\
\text { TOS }\end{array}$ & AUTOR & TITULO & NOMBRE DE LA REVISTA & $\begin{array}{l}\text { AÑO DE } \\
\text { PUBLI- } \\
\text { CACIÓN }\end{array}$ & PAIS & $\begin{array}{l}\text { CON- } \\
\text { TI- } \\
\text { NENTE }\end{array}$ & $\begin{array}{l}\text { SECTOR EM- } \\
\text { PRESARIAL }\end{array}$ \\
\hline 1 & $\begin{array}{l}\text { SCIENCE- } \\
\text { DIRECT }\end{array}$ & $\begin{array}{c}\text { ALFREDO RIVERA, JACOB } \\
\text { KASHIWAGI }\end{array}$ & $\begin{array}{c}\text { IDENTIFYING THE STATE OF THE PROJECT MANAGE- } \\
\text { MENT PROFESSION [6] }\end{array}$ & PROCEDIA ENGINEERING & 2016 & $\begin{array}{l}\text { UNITED } \\
\text { STATES }\end{array}$ & $\begin{array}{l}\text { AME- } \\
\text { RICA }\end{array}$ & TECNOLGIA \\
\hline 2 & $\begin{array}{l}\text { SCIENCE- } \\
\text { DIRECT }\end{array}$ & $\begin{array}{l}\text { KIRSI AALTONEN, TUOMAS } \\
\text { AHOLA, KARLOS ARTTO, }\end{array}$ & $\begin{array}{l}\text { SOMETHING OLD, SOMETHING NEW: PATH DEPEND- } \\
\text { ENCE AND PATH CREATION DURING THE EARLY STAGE } \\
\text { OF A PROJECT[7] }\end{array}$ & $\begin{array}{c}\text { INTERNATIONAL JOURNAL OF PROJECT } \\
\text { MANAGEMENT }\end{array}$ & 2017 & $\begin{array}{l}\text { FIN- } \\
\text { LAND }\end{array}$ & $\begin{array}{l}\text { EU- } \\
\text { ROPA }\end{array}$ & $\begin{array}{l}\text { CONSTRUC- } \\
\text { CION }\end{array}$ \\
\hline 3 & $\begin{array}{l}\text { SCIENCE- } \\
\text { DIRECT }\end{array}$ & $\begin{array}{l}\text { ERIC DANIEL, PIERRE A. } \\
\text { DANIEL, }\end{array}$ & $\begin{array}{c}\text { MEGAPROJECTS AS COMPLEX ADAPTIVE SYSTEMS: } \\
\text { THE HINKLEY POINT C CASE[8] }\end{array}$ & $\begin{array}{l}\text { INTERNATIONAL JOURNAL OF PROJECT } \\
\text { MANAGEMENT, }\end{array}$ & 2019 & FRANCE & $\begin{array}{c}\text { EU- } \\
\text { ROPA }\end{array}$ & $\begin{array}{l}\text { CONSTRUC- } \\
\text { CION }\end{array}$ \\
\hline 4 & $\begin{array}{l}\text { SCIENCE- } \\
\text { DIRECT }\end{array}$ & $\begin{array}{l}\text { H. SANTOS, M.T. PEREIRA, } \\
\text { F.J.G. SILVA, L.P. FE- } \\
\text { RREIRA, }\end{array}$ & $\begin{array}{l}\text { A NOVEl ReWORK COSTING METHODOLOGY AP- } \\
\text { PLIED To a Bus MANUFACTURING COMPANY[9] }\end{array}$ & PROCEDIA MANUFACTURING & 2018 & $\begin{array}{l}\text { PORTU- } \\
\text { GAL }\end{array}$ & $\begin{array}{l}\text { EU- } \\
\text { ROPA }\end{array}$ & INDUSTRIAL \\
\hline 5 & $\begin{array}{l}\text { SCIENCE- } \\
\text { DIRECT }\end{array}$ & $\begin{array}{c}\text { FRANK ZHIGANG FANG, } \\
\text { ZHAOYAN LI, ALAN } \\
\text { AROKIAM, TERRY GOR- } \\
\text { MAN, }\end{array}$ & $\begin{array}{c}\text { CLOSED LOOP PMI DRIVEN DIMENSIONAL QUALITY } \\
\text { LIFECYCLE MANAGEMENT APPROACH FOR SMART } \\
\text { MANUFACTURING SYSTEM[10] }\end{array}$ & PROCEDIA CIRP & 2016 & $\begin{array}{l}\text { UNITED } \\
\text { KING }\end{array}$ & $\begin{array}{l}\text { EU- } \\
\text { ROPA }\end{array}$ & INDUSTRIAL \\
\hline 6 & $\begin{array}{l}\text { SCIENCE- } \\
\text { DIRECT }\end{array}$ & IVANOV NIKOLAY, & $\begin{array}{c}\text { A STUDY ON OPTIMIZATION OF NONCONFORMITIES } \\
\text { MANAGEMENT COST IN THE QUALITY MANAGEMENT } \\
\text { SYSTEM (QMS) OF SMALL-SIZED ENTERPRISE OF THE } \\
\text { CONSTRUCTION INDUSTRY[11] }\end{array}$ & PROCEDIA ENGINEERING & 2016 & RUSIA & $\begin{array}{l}\text { EU- } \\
\text { ROPA }\end{array}$ & INDUSTRIAL \\
\hline 7 & $\begin{array}{l}\text { SCIENCE- } \\
\text { DIRECT }\end{array}$ & $\begin{array}{l}\text { ANDREAS SCHUMACHER, } \\
\text { WILFRIED SIHN, }\end{array}$ & $\begin{array}{c}\text { DEVELOPMENT OF A MONITORING SYSTEM FOR IM- } \\
\text { PLEMENTATION OF INDUSTRIAL DIGITALIZATION AND } \\
\text { AUTOMATION USING } 143 \text { KEY PERFORMANCE INDI- } \\
\text { CATORS[12] }\end{array}$ & PROCEDIA CIRP & 2020 & $\begin{array}{l}\text { AUS- } \\
\text { TRIA }\end{array}$ & $\begin{array}{l}\text { EU- } \\
\text { ROPA }\end{array}$ & INDUSTRIAL \\
\hline 8 & $\begin{array}{l}\text { SCIENCE- } \\
\text { DIRECT }\end{array}$ & $\begin{array}{l}\text { WEIQI XING, JIAN LI HAO, } \\
\text { LIANG QIAN, VIVIAN W.Y. } \\
\text { TAM, KAROL S. SIKORA, }\end{array}$ & $\begin{array}{c}\text { IMPLEMENTING LEAN CONSTRUCTION TECHNIQUES } \\
\text { AND MANAGEMENT METHODS IN CHINESE PROJECTS: } \\
\text { A CASE STUDY IN SUZHOU, CHINA[13] }\end{array}$ & JOURNAL OF CLEANER PRODUCTION & 2021 & CHINA & ASIA & $\begin{array}{l}\text { CONSTRUC- } \\
\text { CION }\end{array}$ \\
\hline 9 & $\begin{array}{l}\text { SCIENCE- } \\
\text { DIRECT }\end{array}$ & $\begin{array}{c}\text { BENYOH EMMANUEL } \\
\text { KIGHA NSAFON, HEMEN } \\
\text { MARK BUTU, ABDULHA- } \\
\text { MEED BABATUNDE } \\
\text { OWOLABI, JONG WOOK } \\
\text { ROH, DONGJUN SUH, } \\
\text { JEUNG-SOO HUH, } \\
\end{array}$ & $\begin{array}{l}\text { INTEGRATING MULTI-CRITERIA ANALYSIS WITH } \\
\text { PDCA CYCLE FOR SUSTAINABLE ENERGY PLANNING } \\
\text { IN AFRICA: APPLICATION TO HYBRID MINI-GRID SYS- } \\
\text { TEM IN CAMEROON [14] }\end{array}$ & $\begin{array}{c}\text { SUSTAINABLE ENERGY TECHNOLOGIES } \\
\text { AND ASSESSMENTS }\end{array}$ & 2020 & $\begin{array}{l}\text { SOUTH } \\
\text { KOREA }\end{array}$ & ASIA & ENERGETICO \\
\hline 10 & $\begin{array}{l}\text { SCIENCE- } \\
\text { DIRECT }\end{array}$ & ANUPAMA PRASHAR, & $\begin{array}{c}\text { ADOPTING PDCA (PLAN-DO-CHECK-ACT) CYCLE } \\
\text { FOR ENERGY OPTIMIZATION IN ENERGY-INTENSIVE } \\
\text { SMES[15] }\end{array}$ & JOURNAL OF CLEANER PRODUCTION & 2017 & INDIA & ASIA & ENERGETICO \\
\hline
\end{tabular}




\begin{tabular}{|c|c|c|c|c|c|c|c|c|}
\hline 11 & SCOPUS & $\begin{array}{l}\text { YU, Y., DUKES, T.J., } \\
\text { SCHMIDT, B.M. }\end{array}$ & $\begin{array}{l}\text { INTEGRATING DESIGN FOR RELIABILITY INTO PROJECT } \\
\text { MANAGEMENT PROCESSES[16] }\end{array}$ & $\begin{array}{l}\text { PROCEEDINGS - ANNUAL RELIABILITY } \\
\text { AND MAINTAINABILITY SYMPOSIUM, } \\
\text { 2019-JANUARY, ART. NO. 8769271, }\end{array}$ & 2019 & $\begin{array}{l}\text { UNITED } \\
\text { STATES }\end{array}$ & $\begin{array}{l}\text { AME- } \\
\text { RICA }\end{array}$ & $\begin{array}{l}\text { ARTICULO DE } \\
\text { FUNDAMEN- } \\
\text { TOS TEORICOS }\end{array}$ \\
\hline 12 & SCOPUS & TARABA, $\mathrm{P}$. & $\begin{array}{c}\text { APPLICATION OF PROJECT MANAGEMENT METHODS IN } \\
\text { THE CZECH REPUBLIC[17] }\end{array}$ & $\begin{array}{l}\text { MATEC WEB OF CONFERENCES, } 210, \\
\text { ART. NO. 02028, }\end{array}$ & 2018 & $\begin{array}{l}\text { CZECH } \\
\text { REPU- } \\
\text { BLIC }\end{array}$ & $\begin{array}{c}\text { EU- } \\
\text { ROPA }\end{array}$ & INDUSTRIAL \\
\hline 13 & SCOPUS & $\begin{array}{l}\text { Koskela, L., TeZEl, A., } \\
\text { PATEL, V. }\end{array}$ & $\begin{array}{l}\text { THEORY OF QUALITY MANAGEMENT: ITS ORIGINS } \\
\text { AND HISTORY[18] }\end{array}$ & $\begin{array}{l}\text { 27TH ANNUAL CONFERENCE OF THE IN- } \\
\text { TERNATIONAL GROUP FOR LEAN CON- } \\
\text { STRUCTION, IGLC 2019, PP. 1381-1390. } \\
\text { CITED } 2 \text { TIMES. }\end{array}$ & 2019 & $\begin{array}{c}\text { IR- } \\
\text { LANDA }\end{array}$ & $\begin{array}{c}\text { EU- } \\
\text { ROPA }\end{array}$ & $\begin{array}{l}\text { ARTICULO DE } \\
\text { FUNDAMEN- } \\
\text { TOS TEORICOS }\end{array}$ \\
\hline 14 & SCOPUS & $\begin{array}{l}\text { BUITRON-LOPEZ, L., } \\
\text { VIACAVA-CAMPOS, G., } \\
\text { EYZAGUIRRE-MUNARRIZ, } \\
\text { J., RAYMUNDO-IBANEZ, C. }\end{array}$ & $\begin{array}{l}\text { LEAN MANUFACTURING MODEL BASED ON THE DEM- } \\
\text { ING CYCLE AND DEVELOPED IN GANTT TO INCREASE } \\
\text { EFFICIENCY IN PLASTIC COMPANIES[19] }\end{array}$ & $\begin{array}{c}\text { IEEE 39TH CENTRAL AMERICA AND } \\
\text { PANAMA CONVENTION, CONCAPAN } \\
\text { 2019, 2019-NOVEMBER, ART. NO. } \\
8976984, . \\
\end{array}$ & 2019 & PERU & $\begin{array}{l}\text { AME- } \\
\text { RICA }\end{array}$ & INDUSTRIAL \\
\hline 15 & SCOPUS & $\begin{array}{l}\text { ZADRY, H.R., DARWIN, R., } \\
\text { ZADRY, H.R. }\end{array}$ & $\begin{array}{c}\text { THE SUCCESS OF 5S AND PDCA IMPLEMENTATION IN } \\
\text { INCREASING THE PRODUCTIVITY OF AN SME IN WEST } \\
\text { SUMATRA[20] }\end{array}$ & $\begin{array}{l}\text { IOP CONFERENCE SERIES: MATERIALS } \\
\text { SCIENCE AND ENGINEERING, } 1003 \text { (1), } \\
\text { ART. NO. 012075, }\end{array}$ & 2020 & $\begin{array}{l}\text { INDONE- } \\
\text { SIA }\end{array}$ & ASIA & INDUSTRIAL \\
\hline 16 & SCOPUS & CHEN, Y., LI, H. & $\begin{array}{l}\text { RESEARCH ON ENGINEERING QUALITY MANAGE- } \\
\text { MENT BASED ON PDCA CYCLE[21] }\end{array}$ & $\begin{array}{l}\text { IOP CONFERENCE SERIES: MATERIALS } \\
\text { SCIENCE AND ENGINEERING, } 490 \text { (6), } \\
\text { ART. NO. 062033, . CITED } 3 \text { TIMES. } \\
\end{array}$ & 2019 & CHINA & ASIA & $\begin{array}{l}\text { CONSTRUC- } \\
\text { CION }\end{array}$ \\
\hline 17 & SCOPUS & $\begin{array}{l}\text { LANGSTON, C., GHAN- } \\
\text { BARIPOUR, A.N. }\end{array}$ & $\begin{array}{l}\text { A MANAGEMENT MATURITY MODEL (MMM) FOR } \\
\text { PROJECT-BASED ORGANIZATIONAL PERFORMANCE AS- } \\
\text { SESSMENT[22] }\end{array}$ & $\begin{array}{l}\text { CONSTRUCTION ECONOMICS AND } \\
\text { BUILDING, } 16 \text { (4), PP. 68-85. CITED } 9 \text { TI- } \\
\text { MES. }\end{array}$ & 2016 & $\begin{array}{l}\text { AUS- } \\
\text { TRIA }\end{array}$ & $\begin{array}{l}\text { OCEA- } \\
\text { NIA }\end{array}$ & $\begin{array}{l}\text { CONSTRUC- } \\
\text { CION }\end{array}$ \\
\hline 18 & SCOPUS & $\begin{array}{l}\text { REN, M.-M., LING, N., } \\
\text { WEI, X., FAN, S.-H. }\end{array}$ & $\begin{array}{l}\text { THE APPLICATION OF PDCA CYCLE MANAGEMENT IN } \\
\text { PROJECT MANAGEMENT[23] }\end{array}$ & $\begin{array}{l}2015 \text { INTERNATIONAL CONFERENCE ON } \\
\text { COMPUTER SCIENCE AND APPLICA- } \\
\text { TIONS, CSA 2015, ART. NO. 7810878, } \\
\text { PP. 268-272. CITED } 6 \text { TIMES. }\end{array}$ & 2017 & CHINA & ASIA & INDUSTRIAL \\
\hline 19 & SCOPUS & $\begin{array}{l}\text { SERRANO, P.S., MERINO, } \\
\text { M.R., SÁEZ, P.V. }\end{array}$ & $\begin{array}{l}\text { METHODOLOGY FOR CONTINUOUS IMPROVEMENT } \\
\text { PROJECTS IN HOUSING CONSTRUCTIONS[24] }\end{array}$ & $\begin{array}{l}\text { BUILDINGS, } 10 \text { (11), ART. NO. } 199 \text {, PP. } 1- \\
\text { 16. CITED } 1 \text { TIME. }\end{array}$ & 2020 & SPAIN & $\begin{array}{l}\text { EU- } \\
\text { ROPA }\end{array}$ & $\begin{array}{l}\text { CONSTRUC- } \\
\text { CION }\end{array}$ \\
\hline 20 & SCOPUS & DOVleAC, R., IONICǍ, A. & $\begin{array}{c}\text { QUALITY MANAGEMENT TECHNIQUES EMBEDDED IN } \\
\text { AGILE PROJECT DEVELOPMENT[25] }\end{array}$ & $\begin{array}{l}\text { MATEC WEB OF CONFERENCES, } 121, \\
\text { ART. NO. 05003, . CITED } 1 \text { TIME. }\end{array}$ & 2017 & $\begin{array}{l}\text { ROMA- } \\
\text { NIA }\end{array}$ & $\begin{array}{c}\text { EU- } \\
\text { ROPA }\end{array}$ & TECNOLOGIA \\
\hline 21 & SCOPUS & $\begin{array}{l}\text { SONG, X.-H., NIU, D.-X., } \\
\text { YE, C.-Q., LU, D.-L. }\end{array}$ & $\begin{array}{c}\text { SURVEY ON THE QUALITY MANAGEMENT SYSTEM OF } \\
\text { POWER DISTRIBUTION PROJECTS BASED ON THE THE- } \\
\text { ORY OF TQM[26] }\end{array}$ & $\begin{array}{l}\text { APPLIED MECHANICS AND MATERIALS, } \\
108, \text { PP. 30-34. }\end{array}$ & 2012 & CHINA & ASIA & ENERGETICO \\
\hline
\end{tabular}


Dada la diversidad de países y sectores empresariales de los artículos seleccionados, los objetivos de cada investigación fueron diversos, como el cumplimiento de cronogramas, la reducción o minimización de costos, mejorar la comprensión de los procesos empresariales, optimizar la planificación, implementación de algoritmos para acciones correctivas entre otros, por lo cual se realizó el análisis de la variable de resumen de los objetivos del proyecto, que cuenta con tres categorías, que corresponde a los puntos comunes de los artículos que fueron: la gestión de proyectos, la gestión de calidad y la gestión de costos.

El $46,2 \%$ de los artículos estaban enfocados en la gestión de proyectos y cumplimiento de cronogramas, mientras el 38,5\% lo estaban a la gestión de calidad, evaluando acciones correctivas que permitieran optimizar procesos que se vieran reflejados en la calidad final de los productos y el 15,4\% se enfocó en la reducción o minimización de costos por medio de la implementación del ciclo PHVA. La Tabla 4 muestra el objetivo, el tema y los resultados de cada documento analizado.

Excluyendo dos artículos con un enfoque netamente teórico, que sin embargo brinda datos sobre las bondades del ciclo PHVA en la mejora continua en las fases de la gestión de proyectos, el $100 \%$ de las investigaciones reflejaron un impacto positivo en los objetivos planteados posterior a la implementación del ciclo PHVA.

Tabla 4. Objetivos y resultados de los 21 artículos seleccionados en la revisión bibliográfica.

\begin{tabular}{|c|c|c|c|c|c|}
\hline $\mathrm{No}$ & AUTOR & TITULO & OBJETIVO & TEMA & RESULTADO \\
\hline 1 & $\begin{array}{l}\text { ALFREDO RI- } \\
\text { VERA, JACOB } \\
\text { KASHIWAGI }\end{array}$ & $\begin{array}{l}\text { IDENTIFYING THE } \\
\text { STATE OF THE PRO- } \\
\text { JECT MANAGEMENT } \\
\text { PROFESSION [6] }\end{array}$ & $\begin{array}{l}\text { RESUMIR LAS METODOLOGÍAS } \\
\text { DE GESTIÓN DE PROYECTOS } \\
\text { (PM) MÁS UTILIZADAS EN EL } \\
\text { MUNDO, }\end{array}$ & $\begin{array}{l}\text { GES- } \\
\text { TIÓN DE } \\
\text { PROYEC- } \\
\text { TOS }\end{array}$ & $\begin{array}{c}\text { DESEMPEÑO COMPROBADO EN MÁS DEL 90\% DE SUS } \\
\text { PROYECTOS, UNA REDUCCIÓN DE LOS COSTOS EN } \\
\text { PROMEDIO DEL } 31 \% \text { Y UNA SATISFACCIÓN DEL } \\
\text { CLIENTE DEL } 98 \% \text {. }\end{array}$ \\
\hline 2 & $\begin{array}{l}\text { KIRSI AALTONEN, } \\
\text { TUOMAS AHOLA, } \\
\text { KARLOS ARTTO, }\end{array}$ & $\begin{array}{c}\text { SOMETHING OLD, } \\
\text { SOMETHING NEW: } \\
\text { PATH DEPENDENCE } \\
\text { AND PATH CREATION } \\
\text { DURING THE EARLY } \\
\text { STAGE OF A PRO- } \\
\text { JECT[7] } \\
\end{array}$ & $\begin{array}{l}\text { AUMENTAR LA COMPRENSIÓN } \\
\text { DE LOS PROCESOS DE DEPEN- } \\
\text { DENCIA Y CREACIÓN DE CAMI- } \\
\text { NOS DURANTE LA ETAPA INI- } \\
\text { CIAL DEL PROYECTO. }\end{array}$ & $\begin{array}{l}\text { GES- } \\
\text { TIÓN DE } \\
\text { PROYEC- } \\
\text { TOS }\end{array}$ & MEJORAS EN LA EJECUCIÓN DE PROYECTOS \\
\hline 3 & $\begin{array}{l}\text { ERIC DANIEL, } \\
\text { PIERRE A. DAN- } \\
\text { IEL, }\end{array}$ & $\begin{array}{l}\text { MEGAPROJECTS AS } \\
\text { COMPLEX ADAPTIVE } \\
\text { SYSTEMS: THE HIN- } \\
\text { KLEY POINT C CASE[8] }\end{array}$ & $\begin{array}{c}\text { COMPRENDER Y MODELAR } \\
\text { LOS PROCESOS DE EVOLUCIÓN } \\
\text { EN EL MEGAPROYECTO DE LA } \\
\text { CENTRAL NUCLEAR DE HIN- } \\
\text { KLEY POINT C. } \\
\end{array}$ & $\begin{array}{l}\text { GES- } \\
\text { TIÓN DE } \\
\text { PROYEC- } \\
\text { TOS }\end{array}$ & $\begin{array}{l}\text { AUMENTO EN EL CUMPLIMIENTO DE LOS CRONOGRA- } \\
\text { MAS ESTABLECIDOS EN EL MEGAPROYECTO DE HASTA } \\
\text { EL 20\% }\end{array}$ \\
\hline 4 & $\begin{array}{l}\text { H. SANTOS, M.T. } \\
\text { PEREIRA, F.J.G. } \\
\text { SILVA, L.P. FE- } \\
\text { RREIRA, }\end{array}$ & $\begin{array}{l}\text { A NOVEL REWORK } \\
\text { COSTING METHODOL- } \\
\text { OGY APPLIED TO A } \\
\text { BuS MANUFACTURING } \\
\text { COMPANY[9] }\end{array}$ & $\begin{array}{c}\text { MEJORAR EL INDICADOR DE } \\
\text { COSTOS DE CALIDAD, PROPOR- } \\
\text { CIONANDO MEDIOS PARA EVA- } \\
\text { LUAR EL COSTO DE FALLA } \\
\text { DENTRO DEL PROCESO PRO- } \\
\text { DUCTIVO DE LA INDUSTRIA } \\
\text { AUTOMOTRIZ. } \\
\end{array}$ & $\begin{array}{l}\text { GES- } \\
\text { TIÓN } \\
\text { CALI- } \\
\text { DAD- } \\
\text { COSTOS }\end{array}$ & $\begin{array}{l}\text { MEJORO EL FUNCIONAMIENTO COMO UNA HERRA- } \\
\text { MIENTA PARA MEDIR LOS COSTOS DE DESPERDICIO. }\end{array}$ \\
\hline 5 & $\begin{array}{l}\text { FRANK ZHIGANG } \\
\text { FANG, ZHAOYAN } \\
\text { LI, ALAN } \\
\text { AROKIAM, TERRY } \\
\text { GORMAN, }\end{array}$ & $\begin{array}{l}\text { CLOSED LOOP PMI } \\
\text { DRIVEN DIMENSIONAL } \\
\text { QUALITY LIFECYCLE } \\
\text { MANAGEMENT AP- } \\
\text { PROACH FOR SMART } \\
\text { MANUFACTURING } \\
\text { SYSTEM[10] } \\
\end{array}$ & $\begin{array}{l}\text { PHVA PARTIR DEL DISEÑO DI- } \\
\text { MENSIONAL, LA PLANIFICA- } \\
\text { CIÓN Y LAS INSPECCIONES A } \\
\text { TRAVÉS DE UN REPOSITORIO } \\
\text { DE DATOS ÚNICO }\end{array}$ & $\begin{array}{l}\text { GES- } \\
\text { TIÓN DE } \\
\text { PROYEC- } \\
\text { TOS }\end{array}$ & $\begin{array}{l}\text { PERMITIR QUE EL DISEÑO PARA LA FABRICACIÓN LO- } \\
\text { GRE UN EQUILIBRIO ÓPTIMO DE COSTO / RENDI- } \\
\text { MIENTO EN UNA ETAPA MUY TEMPRANA DEL PRO- } \\
\text { YECTO. }\end{array}$ \\
\hline 6 & $\begin{array}{l}\text { IVANOV NIKO- } \\
\text { LAY, }\end{array}$ & $\begin{array}{l}\text { A STUDY ON OPTIMI- } \\
\text { ZATION OF NONCON- } \\
\text { FORMITIES MANAGE- } \\
\text { MENT COST IN THE }\end{array}$ & $\begin{array}{l}\text { IMPLEMENTAR ALGORITMOS } \\
\text { DE CLASIFICACIÓN ORIGINAL } \\
\text { DE ACCIONES CORRECTIVAS } \\
\text { CON EL PROPÓSITO DE MINIMI- }\end{array}$ & $\begin{array}{l}\text { MINIMI- } \\
\text { ZAR } \\
\text { COSTOS }\end{array}$ & $\begin{array}{l}\text { EL RESULTADO DEL ALGORITMO GENERO DISMINU- } \\
\text { CIÓN EN LOS COSTOS DE ELIMINACIÓN DE LOS PRO- } \\
\text { DUCTOS NO CONFORMES DE LAS EMPRESAS QUE LO } \\
\text { IMPLEMENTARON }\end{array}$ \\
\hline
\end{tabular}


QUALITY MANAGEMENT SYSTEM (QMS) OF SMALL-SIZED ENTERPRISE OF THE CONSTRUCTION INDUSTRY[11]

DEVELOPMENT OF A

MONITORING SYSTEM

ANDREAS SCHUFOR IMPLEMENTATION 7 MACHER, WILFRIED SIHN, OF INDUSTRIAL DIGITALIZATION AND AUTOMATION USING 143 KEY PERFORMANCE INDICATORS[12]

IMPLEMENTING LEAN CONSTRUCTION TECH-

WEIQI XING, JIAN

LI HAO, LIANG

QIAN, VIVIAN

W.Y. TAM,

KAROL S. SIKORA,

NIQUES AND MANAGE-

MENT METHODS IN

CHINESE PROJECTS: A

CASE STUDY IN SUZHOU, CHINA[13]

BENYOH EMMANUEL KIGHA

NSAFON, HEMEN

MARK BUTU, ABDULHAMEED BABATUNDE

OWOLABI, JONG

WOOK ROH,

DONGJUN SUH, JEUNG-SOO HuH,
INTEGRATING MULTIWITH PDCA CYCLE FOR SUSTAINABLE ENERGY PLANNING IN AFRICA: APPLICATION TO HYBRID MINI-GRID SYSTEM IN CAMEROON [14] CRITERIA ANALYSIS
ZAR LOS COSTOS POTENCIA-

LES DE ELIMINACIÓN DE LOS

POSIBLES PRODUCTOS NO CONFORMES

PROPONER UN SISTEMA DE SE- GESGUIMIENTO HOLÍSTICO, UTILIZANDO 143 INDICADORES CLAVE DE DESEMPEÑO KPÌ TIÓN DE PROYEC-

TOS

IMPLEMENTACIÓN DE LC (LEAN CANVAS) EN UN PROYECTO DE CONSTRUCCIÓN INDUSTRIAL CHINO PARA LLENAR UN VACÍO DE CONOCIMIENTO.
GES-

TIÓN DE

PROYEC-

TOS
LA GENERACIÓN DEL SISTEMA DE MONITOREO RESULTO EN UNA MEJORÍA EN TODAS LAS DIMENSIONES EVALUADAS ESTRATEGIA Y LIDERAZGO, ATENCIÓN AL CLIENTE, PROCESOS, CONTROL DE CALIDAD, LOGÍSTICA, COMPRAS Y CIBERSEGURIDAD.
INTEGRAR AHP-VIKOR CON EL CICLO PDCA, PARA LA MEJORA CONTINUA DEL PROCESO DE PLANIFICACIÓN DE UN PLAN DE INSTALACIÓN DE ENERGÍA RENOVABLE EN ÁFRICA
GESTIÓN DE PROYECTOS

\subsection{1}

PROPORCIONAR ORIENTACIÓN TANTO TEÓRICA COMO PRÁCTICA A LAS EMPRESAS CONSTRUCTORAS QUE PRETENDEN IMPLEMENTAR LCM (MODELO DE CAMBIO APLICANDO VALORES AGILES) EN PROYECTOS FUTUROS.

LOS RESULTADOS MUESTRAN QUE EL SISTEMA DE ENERGÍA HÍBRIDA SELECCIONADO NO SOLO GARANTIZA UNA CONFIGURACIÓN CONFIABLE DEL SISTEMA CON UNA CARGA INSATISFECHA DE $2.45 \mathrm{KWH} /$ AÑO, SINO QUE TAMBIÉN TIENE BENEFICIOS ECONÓMICOS SOBRE EL SISTEMA DE MENOR COSTO Y UN SISTEMA DE GENERADOR DIESEL ESTÁNDAR RESPECTIVAMENTE: INGRESOS POR VENTAS DE ENERGÍA DE 3.816.7 \$ / AÑO Y 745,11 \$ / AÑO; Y AHORROS POR EL PRECIO DEL CARBONO DE 68,810.17 \$ / AÑO Y $8,323,774.7$ \$ AÑO.

EL ANÁLISIS DE CASOS MOSTRÓ UNA REDUCCIÓN DEL

ADOPTING PDCA

(PLAN-DO-CHECK-

ANUPAMA PRAS- ACT) CYCLE FOR EN- ENERGÉTICA CONSTANTE Y HAR,

ERGY OPT ENERGY-INTENSIVE SMES[15]

LOGRAR UNA EFICIENCIA ENERGÉTICA CONSTANTE Y
UNA PRODUCCIÓN MÁS LIMPIA EN LAS PYMES.
GESTIÓN

CALI-

DAD
ANALIZAR LA ADMINISTRACIÓN DE CONFIABILIDAD DESDE LA PERSPECTIVA DEL CICLO DE VIDA DEL PROYECTO

DETERMINAR EL NIVEL DE APLICACIÓN DE LOS MÉTODOS DE GESTIÓN DE PROYECTOS EN LA REPÚBLICA CHECA ENTRE LOS AÑOS 2015 A 2017. DETERMINAR LOS FUNDAMENTOS TEÓRICOS Y FILOSÓFICOS DE LA GESTIÓN DE LA CALIDAD, SEGÚN HAN IDO

GESTIÓN DE PROYECTOS

GESTIÓN DE PROYECTOS

GESTIÓN

CALIDAD

\section{$35 \% \mathrm{EN}$ \\ $35 \%$ EN}

CONSUMO DE ENERGÍA (SEC) DE UNO DE LOS SISTEMAS DE VACÍO DE LA MÁQUINA DE PAPEL (PM) MEDIANTE LA IMPLEMENTACIÓN DE ESA PARA LA OPTIMIZACIÓN DEL VACÍO, GENERANDO UN AHORRO ENERGÉTICO ANUAL DE 0,3 GJ / Y UN AHORRO ADICIONAL DE COSTOS DE ENERGÍA DE USD 26,900.

SE CREAN TRES PROCESOS PROPUESTOS EN EL ÁREA DE CONOCIMIENTO DE LA CONFIABILIDAD BASADOS EN LOS DOS ESTÁNDARES DE CONFIABILIDAD MÁS REFERENCIADOS EN LA ACTUALIDAD:

EL ESTUDIO DEMOSTRÓ UN AUMENTO EN LA APLICACIÓN DE MÉTODOS DE GESTIÓN DE PROYECTOS EN LAS INDUSTRIAS DEL PAÍS, DURANTE EL PERIODO DE ESTUDIO.

GESTIÓN DE CALIDAD PARA EL CUMPLIMIENTO DE METAS 
EVOLUCIONANDO Y CAM-

BIANDO A LO LARGO DEL TIEMPO.

BUITRON-LOPEZ, LEAN MANUFACTUR-

L., VIACAVA- ING MODEL BASED ON

CAMPOS, G.,

14 EYZAGUIRRE-

MUNARRIZ, J., GANTT TO INCREASE

RAYMUNDO-IBA- EFFICIENCY IN PLASTIC NEZ, C. COMPANIES[19]

THE SUCCESS OF 5S AND PDCA IMPLE-

ZADRY, H.R.,

15 DARWIN, R.,

ZADRY, H.R. MENTATION IN INCREASING THE

PRODUCTIVITY OF AN SME IN WEST SUMATRA[20]

RESEARCH ON ENGINEERING QUALITY

16 CHEN, Y., LI, H.

MANAGEMENT BASED ON PDCA CYCLE[21]

17 GHANBARIPOUR, A.N. BASED ORGANIZA-
DESARROLLAR UNA ESTRATEGIA QUE AUMENTE LA EFICIENCIA DE LAS MÁQUINAS DE MANERA SOSTENIDA.

IMPLEMENTAR LA METODOLOGÍA 5S, A UNA PYME X DE PADANG, EN EL OESTE DE SUMATRA, QUE SE DEDICA A LA FABRICACIÓN DE ZAPATOS HECHOS A MANO.

GESTIONAR LA CALIDAD EN LA CONSTRUCCIÓN Y REDUCCIÓN APLICA LOS PRINCIPIOS DEL CICLO PDCA A LA GESTIÓN DE LA CALIDAD DEL PROYECTO

DESARROLLAR Y ANALIZAR UN MODELO DE MADUREZ DE

A MANAGEMENT MA- LA GESTIÓN (MMM) PARA TURITY MODEL EVALUAR LA MADUREZ DE

(MMM) FOR PROJECT- LAS ORGANIZACIONES DE GESTIONAL PERFORMANCE ASSESSMENT[22]

REN, M.-M., 18 LING, N., WEI, X., FAN, S.-H.

THE APPLICATION OF PDCA CYCLE MANAGEMENT IN PROJECT MANAGEMENT[23]

\section{TIÓN DE PROYECTOS A TRA-}

VÉS DE UNA METODOLOGÍA PERSONALIZABLE, SISTEMÁTICA, ESTRATÉGICA Y PRÁCTICA.

ANALIZAR DETALLADAMENTE

LA GESTIÓN DEL CICLO DE

PDCA EN LA EN LA GESTIÓN DEL CRONOGRAMA DEL PROYECTO, LA GESTIÓN DE LA CALIDAD Y LA GESTIÓN DE COSTOS DE INGENIERÍA EN EL MODO DE OPERACIÓN.
GES-

TIÓN DE

PROYECTOS
GES-

TIÓN

CALI-

DAD
GESTIÓN DE PROYECTOS-CALIDAD
LA IMPLEMENTACIÓN DE LA PROPUESTA HACIENDO USO DE FILOSOFÍA KAIZEN, LA METODOLOGÍA'LAS 5S Y TPM PLANEADAS SE TRADUCEN EN MEJORAS SIGNIFICATIVAS PARA EL INCREMENTO EN LA EFICIENCIA DE 60\&\% EN 2017 A 67\% EN 2019 Y DISMINUCIÓN DE LOS RECLAMOS DE CLIENTES DEL $90 \%$ AL 95\%B EN EL MISMO PERIODO

GESTIÓN

CALI-

DAD

DESPUÉS DE UN MES DE APLICACIÓN HUBO DISMINUCIÓN EN LOS PRODUCTOS DEFECTUOSOS DEL 12\% AL $0 \%$ POR MES. Y UNA REDUCCIÓN DE LAS PÉRDIDAS POR PRODUCTOS DE CALZADO DEFECTUOSOS, QUE ANTERIORMENTE ERAN DE RP. 1.250.000 POR SEMANA A CERO

EL AUTOR LLEGA A LA CONCLUSIÓN QUE INDEPENDIENTEMENTE DE LA ETAPA O DEL PROCESO COMPLETO EN EL QUE SE ENCUENTRE EL PROYECTO, SE DEBE APLICAR EL PRINCIPIO DEL CICLO PDCA PARA LOGRAR EL NIVEL ÓPTIMO DE GESTIÓN DE LA CALIDAD DEL PROYECTO.
ENTREGAR EL MODELO MMM QUE ES UN MODELO DE MADUREZ BASADO EN CICLO DE MEJORA CONTINUA PDCA INTEGRADO CON EN LA METODOLOGÍA PMBOK, PARA EVALUAR LA MADUREZ Y EL DESEMPEÑO DE LAS ORGANIZACIONES EN ENTORNOS DE GESTIÓN DE PROYECTO
GESTIÓN DE PROYEC-

TOS TOS

SE LOGRO UNA DISMINUCIÓN EN RIESGO DE CAMBIOS DE COSTOS E IDENTIFICACIÓN Y ANÁLISIS, PARA LA TOMA DE DECISIONES DEL PROYECTO, LA REDUCCIÓN DE LOS COSTOS DEL PROYECTO Y LOS ARREGLOS RAZONABLES PARA EL CRONOGRAMA IMPLEMENTADOS DE MANERA EFECTIVA.

LA IMPLEMENTACIÓN DEL PROCEDIMIENTO LOGRÓ REDUCIR ALREDEDOR DE UN 45\% LOS COSTOS DERIVADOS DE LOS DEFECTOS OCASIONADOS DURANTE LA EJECUCIÓN DEL REVESTIMIENTO CERÁMICO, DEBIDO

METHODOLOGY FOR CONTINUOUS IM-

SERRANO, P.S., 19 MERINO, M.R., SÁEZ, P.V.
PROVEMENT PROJECTS IN HOUSING CONSTRUCTIONS[24]
EVIDENCIAR UN PROCEDIMIENTO BASADO EN LA MEJORA CONTINUA DE PROYECTOS PARA UTILIZAR EN CONSTRUCCIÓN DE EDIFICIOS
GES-

TIÓN

CALI-

DAD

\begin{abstract}
A LAS MEJORES PRÁCTICAS INCORPORADAS ENEL
\end{abstract} PROCEDIMIENTO

TRAVÉS DE ESTE PROCESO ITERATIVO, PARA MEJORAR GRADUALMENTE LA CALIDAD DEL PROYECTO, REDUCIR LOS COSTOS DEL PROYECTO Y LOS ARREGLOS RAZONABLES PARA EL CRONOGRAMA IMPLEMENTADOS DE MANERA EFECTIVA. 
DOVLEAC, R., IONICĂ, A.
QUALITY MANAGE-

MENT TECHNIQUES EMBEDDED IN AGILE

PROJECT DEVELOPMENT[25]
ANALIZAR EL ENFOQUE AGILE Y LAS HERRAMIENTAS UTILIZADAS PARA GARANTIZAR LA

CALIDAD EN CADA ETAPA DEL CICLO DE VIDA DEL DESARROLLO DE UN PRODUCTO TECNOLÓGICO

CONSTRUIR UN MODELO DE SISTEMA BÁSICO PARA LA

SonG, X.-H.,

21 NIU, D.-X., YE, C.-Q., LU, D.-L.
SURVEY ON THE QUALITY MANAGEMENT SYSTEM OF POWER DISTRIBUTION PROJECTS BASED ON THE THEORY OF TQM[26]
GESTIÓN DE LA CALIDAD EN

LA INGENIERÍA DE DISTRIBUCIÓN DE ENERGÍA AL COMBINAR LOS PUNTOS BÁSICOS DE TQM
ENTREGA DE UN MODELO DE GESTIÓN DEL PROCESO DE PRODUCCIÓN DE PROYECTOS QUE COMBINA LA CALIDAD MONITOREADA Y ENTREGADA EN CADA ETAPA DEL PROYECTO CON LA AYUDA DE LA COMBINACIÓN DE LAS HERRAMIENTAS TRADICIONALES DE GESTIÓN DE LA CALIDAD INTEGRADAS CON LAS HERRAMIENTAS DE CREATIVIDAD MODERNAS CON EL USO DEL PCDA, EL CONTROL DE CALIDAD DEL PROYECTO DE INGENIERÍA PUEDE REALIZAR UNA VERIFICACIÓN SERIA DESDE CADA PROCEDIMIENTO HASTA EL PROYECTO SUBDIVISIONAL, DIVIDIDO Y UNITARIO, PARA MEJORAR LA CALIDAD EN TODOS LOS NIVELES Y LOGRAR LOS ESTÁNDARES Y REQUISITOS DE CALIDAD FINALES.

\section{DISCUSIÓN}

En la actualidad existe un rápido crecimiento de conocimiento científico intensificado por el alto volumen de publicaciones electrónicas en internet, lo cual hace necesario desarrollar estudios que permitan a los profesionales de todas a las áreas de conocimiento, investigaciones que permitan el acceso a resúmenes de información de calidad en temas específicos [1], razón por la cual, la presente investigación desarrolló una búsqueda estructurada metodológicamente utilizando palabras claves, conectores booleanos y criterios de tipificación para la selección de los mejores estudios en un tema trascendental como la implementación del ciclo PHVA en la mejora continua de gestión de proyectos.

Teniendo en cuenta que la realización de revisiones de la literatura científica permite tener un conocimiento actualizado sobre un tema de interés. La presente investigación se podría caracterizar como una revisión narrativa, reflexiva, documental o descriptiva, que proporciona al lector la oportunidad de actualizarse en diversos temas con la enorme cantidad de literatura que se publica a diario, teniendo en cuenta que según datos bibliométricos la ciencia se renueva un alto porcentaje en solo 3 años.

En la literatura científica la calidad de una revista, se mide a través de indicadores de popularidad y prestigio, este es un concepto multidimensional en el que pueden intervenir varios factores como hábitos de citación, tamaño de la comunidad científica, tiempo, etc. En un estudio bibliométrico donde se incluyó Scopus y Web Of Science se encontró alta correlación encontrada entre diversos indicadores de calidad, concluyendo que ambas son fuentes confiables y de amplio espectro para la búsqueda de literatura científica [27]. Por su parte ScienceDirect es una base de datos de revistas con índices de calidad elevados, con altos volúmenes de evidencia, que tiende a crecer en el tiempo [28]

La importancia de la calidad para la competitividad de las empresas es innegable; la función de calidad dentro de las organizaciones, su impacto en el mercado y el aumento en el interés de la comunidad académica, ha provocado un crecimiento en los conceptos de calidad [29], donde el ciclo PHVA ha tenido un papel trascendental desde que fuera propuesto por Deming.

Una de las bondades de la implementación del ciclo PHVA radica en la gestión de proyectos, donde promueve proyectos eficientes y de alta calidad [6]. Encontrando algunos estudios donde es crucial la gestión de proyectos sobre todo en las etapas iniciales del mismo, donde se presentan un alto volumen de dificultades, cuyo rezago afecta el cumplimiento de una adecuada conclusión del proyecto [7]. La pluralidad del ciclo PHVA en la mejora continua de la gestión de proyectos, se evidencia por su versatilidad en el uso de megaproyectos como el de la Central Nuclear de Hinkley Point C [8]. 
El impacto positivo del ciclo PHVA se ve reflejado en la reducción u optimización de costos como se evidencia en H. Santos [9] e Ivanov Nikolay [11], donde se reflejó la utilidad en la toma de decisiones para optimizar los costos empresariales.

El Ciclo PHVA en la mejora continua de la gestión de proyectos y la Calidad fue reportada por más del $80 \%$ de los estudios seleccionados, encontrando en todos los estudios resultados positivos, en diversos sectores empresariales y países, evidenciando la versatilidad del ciclo PHVA que propone desde enfoques holísticos hasta la Digitalización y Automatización Industrial (DA), impulsada a través de nuevos paradigmas productivos como la Industria 4.0 [8], [10], [12]-[15], [17], [18]

En el Continente Europeo se encontró el mayor volumen de publicación sobre la implementación del ciclo PHVA en la Mejora continua de la gestión de proyectos, lo cual coincide con estudios bibliométricos realizados en temas de calidad, comparando la producción por continentes y países, donde se ha reportado que el continente europeo lidera las publicaciones, debido a que la mayoría de sus países aporta en ello [30].

Los mayores sectores fueron el sector industrial y de la construcción, lo cual resulta coherente teniendo en cuenta que son sectores muy grandes y con presencia mundial, además fue en la industria donde surgieron los procesos modernos de calidad y existe una cultura de calidad [31].

Entre las limitantes de la presente investigación se tiene la búsqueda solo en dos bases de datos como fueron Scopus y ScienceDirect. Sin embargo, estas relacionan altos volúmenes de evidencia científica, en cientos de revistas y millones de publicaciones. Otra limitante detectada fue el idioma, donde se omitió el Portugués que podría haber aportado en la revisión de publicaciones por continente, porque previamente se estableció el criterio de búsqueda de publicaciones en inglés teniendo en cuenta la mayoría de las publicaciones presentes en las bases de datos empleadas para la búsqueda.

Otra limitante detectada fue no incluir algún formato o escala de lectura crítica para la selección de los artículos con mejor nivel de evidencia, sin embargo, estos apartados fueron revisados en la etapa final de selección de literatura, por parte del investigador evaluando la coherencia del objetivo planteado, con la estructura metodológica y los resultados.

El autor de la presente investigación declara no tener ningún conflicto de interés relacionados con la selección de los artículos sobre la implementación del ciclo PHVA en la mejora continua de la gestión de proyectos y que la financiación se realizó de manera independiente con recursos propios.

\section{CONCLUSIONES}

Se concluye que frente al tema de calidad existe un alto volumen de literatura, puesto que, a pesar de plantear una estrategia de búsqueda específica, se recogieron casi 200 resultados, que debieron ser depurados. Además, el tema del ciclo PHVA en la mejora continua de la gestión de proyectos, es vigente y actual, puesto que entre 2016 y 2020 se encontraron el mismo número de artículos publicados en el tema de investigación.

Se concluye que la calidad metodológica de los artículos debe ser revisada, dado que se hallaron falencias y debilidades metodológicas en cerca de 50 artículos ya publicados en revistas indexadas, donde no se espera encontrar dichas inconsistencias.

Latinoamérica debe iniciar un proceso de investigación en temas relacionados con la calidad y aun mejor con el ciclo PHVA, dado que solo se encontró un estudio, realizado en Perú. Por continentes se encontró que Europa lideró el número de publicaciones con más de la mitad del total seguida por el continente asiático.

Finalmente se concluye en base a la literatura consultada, que la aplicación del ciclo PHVA en la gestión de proyectos, tiene gran incidencia en la mejora continua de los procesos de gestión que enfrenta todo tipo de proyecto, como lo demuestran los resultados del estudio realizado en china, donde se analizó detalladamente 
la gestión del ciclo de Deming en la gestión del cronograma del proyecto, la gestión de la calidad y la gestión de costos de ingeniería en el modo de operación, [23]. arrojando como resultado final, un procedimiento iterativo, para la construcción de la gestión de los procesos enunciados, provocando la disminución en riesgos de cambios, que permiten realizar la identificación y análisis de la gestión del proyecto, para la toma de decisiones que conlleven a la reducción de los costos del proyecto, así como a ajustes razonables del cronograma, que pueden implementarse de manera efectiva.

\section{AGRADECIMIENTOS}

Se hace un reconocimiento a la Universidad Militar Nueva Granada, sede Campus y en específico al programa de Maestría en Gerencia de Proyectos por el fomento al proceso de investigación para el beneficio de la comunidad académica y las organizaciones del sector real.

\section{REFERENCIAS BIBLIOGRÁFICAS}

[1] G. Goris y S. J. Adolf, «Utilidad y tipos de revisión de literatura», Ene, vol. 9, n.o 2, pp. 0-0, 2015, doi: 10.4321/S1988-348X2015000200002.

[2] B. R. Barringer, F. F. Jones, y D. O. Neubaum, «A quantitative content analysis of the characteristics of rapid-growth firms and their founders», J. Bus. Ventur., vol. 20, n.o 5, pp. 663-687, sep. 2005, doi: 10.1016/j.jbusvent.2004.03.004.

[3] J. M. Morán, A. Santillán-García, y I. Herrera-Peco, «SCRUTATIOm: cómo detectar literatura retractada incluida en revisiones sistemáticas y metaanálisis usando SCOPUS@ y ZOTERO@», Gac. Sanit., ago. 2020, doi: 10.1016/j.gaceta.2020.06.012.

[4] Journal of Cleaner Production. Accedido: may 18, 2021. [En línea]. Disponible en: https://www.journals.elsevier.com/journal-of-cleaner-production

[5] Procedia CIRP. Accedido: may 18, 2021. [En línea]. Disponible en: https://www.journals.elsevier.com/procedia-cirp

[6] A. Rivera y J. Kashiwagi, «Identifying the State of the Project Management Profession», Procedia Eng., vol. 145, pp. 1386-1393, 2016, doi: 10.1016/j.proeng.2016.04.204.

[7] K. Aaltonen, T. Ahola, y K. Artto, «Something old, something new: Path dependence and path creation during the early stage of a project», Int. J. Proj. Manag., vol. 35, n.o 5, pp. 749-762, jul. 2017, doi: 10.1016/j.ijproman.2017.03.004.

[8] E. Daniel y P. A. Daniel, «Megaprojects as complex adaptive systems: The Hinkley point C case», Int. J. Proj. Manag., vol. 37, n.o 8, pp. 1017-1033, nov. 2019, doi: 10.1016/j.ijproman.2019.05.001.

[9] H. Santos, M. T. Pereira, F. J. G. Silva, y L. P. Ferreira, «A Novel Rework Costing Methodology Applied To a Bus Manufacturing Company», Procedia Manuf., vol. 17, pp. 631-639, 2018, doi: 10.1016/j.promfg.2018.10.109.

[10] F. Z. Fang, Z. Li, A. Arokiam, y T. Gorman, «Closed Loop PMI Driven Dimensional Quality Lifecycle Management Approach for Smart Manufacturing System», Procedia CIRP, vol. 56, pp. 614-619, 2016, doi: 10.1016/j.procir.2016.10.121.

[11] I. Nikolay, «A Study on Optimization of Nonconformities Management Cost in the Quality Management System (QMS) of Small-sized Enterprise of the Construction Industry», Procedia Eng., vol. 153, pp. 228-231, 2016, doi: 10.1016/j.proeng.2016.08.107. 
[12] A. Schumacher y W. Sihn, «Development of a Monitoring System for Implementation of Industrial Digitalization and Automation using 143 Key Performance Indicators», Procedia CIRP, vol. 93, pp. 1310-1315, 2020, doi: 10.1016/j.procir.2020.03.012.

[13] W. Xing, J. L. Hao, L. Qian, V. W. Y. Tam, y K. S. Sikora, «Implementing lean construction techniques and management methods in Chinese projects: A case study in Suzhou, China», J. Clean. Prod., vol. 286, p. 124944, mar. 2021, doi: 10.1016/j.jclepro.2020.124944.

[14] B. E. K. Nsafon, H. M. Butu, A. B. Owolabi, J. W. Roh, D. Suh, y J.-S. Huh, «Integrating multi-criteria analysis with PDCA cycle for sustainable energy planning in Africa: Application to hybrid mini-grid system in Cameroon», Sustain. Energy Technol. Assess., vol. 37, p. 100628, feb. 2020, doi: 10.1016/j.seta.2020.100628.

[15] A. Prashar, «Adopting PDCA (Plan-Do-Check-Act) cycle for energy optimization in energyintensive SMEs», J. Clean. Prod., vol. 145, pp. 277-293, mar. 2017, doi: 10.1016/j.jclepro.2017.01.068.

[16] Y. Yu, T. J. Dukes, y B. M. Schmidt, «Integrating Design for Reliability into Project Management Processes», en 2019 Annual Reliability and Maintainability Symposium (RAMS), Orlando, FL, USA, ene. 2019, pp. 1-6. doi: 10.1109/RAMS.2019.8769271.

[17] P. Taraba, «Application of project management methods in the Czech Republic», MATEC Web Conf., vol. 210, p. 02028, 2018, doi: 10.1051/matecconf/201821002028.

[18] L. Koskela, A. Tezel, y V. Patel, «Theory of Quality Management: Its Origins and History», Dublin, Ireland, jul. 2019, pp. 1381-1390. doi: 10.24928/2019/0259.

[19] L. Buitron-Lopez, G. Viacava-Campos, J. Eyzaguirre-Munarriz, y C. Raymundo-Ibanez, «Lean Manufacturing model based on the Deming cycle and developed in Gantt to increase efficiency in plastic companies», en 2019 IEEE 39th Central America and Panama Convention (CONCAPAN XXXIX), Guatemala City, Guatemala, nov. 2019, pp. 1-5. doi: 10.1109/CONCAPANXXXIX47272.2019.8976984.

[20] H. R. Zadry y R. Darwin, «The Success of 5S and PDCA Implementation in Increasing the Productivity of an SME in West Sumatra», IOP Conf. Ser. Mater. Sci. Eng., vol. 1003, p. 012075, dic. 2020, doi: 10.1088/1757-899X/1003/1/012075.

[21] Y. Chen y haoqi Li, «Research on Engineering Quality Management Based on PDCA Cycle», IOP Conf. Ser. Mater. Sci. Eng., vol. 490, p. 062033, abr. 2019, doi: 10.1088/1757899X/490/6/062033.

[22] C. Langston y A. N. Ghanbaripour, «A Management Maturity Model (MMM) for projectbased organisational performance assessment», Constr. Econ. Build., vol. 16, n.o 4, pp. 68-85, dic. 2016, doi: 10.5130/AJCEB.v16i4.5028.

[23] M.-M. Ren, N. Ling, X. Wei, y S.-H. Fan, «The Application of PDCA Cycle Management in Project Management», en 2015 International Conference on Computer Science and Applications (CSA), Wuhan, China, nov. 2015, pp. 268-272. doi: 10.1109/CSA.2015.84.

[24] P. del Solar Serrano, M. del Río Merino, y P. Villoria Sáez, «Methodology for Continuous Improvement Projects in Housing Constructions», Buildings, vol. 10, n.o 11, p. 199, nov. 2020, doi: 10.3390/buildings10110199.

[25] R. Dovleac y A. Ionică, «Quality Management techniques embedded in Agile Project Development», MATEC Web Conf., vol. 121, p. 05003, 2017, doi: 10.1051/matecconf/201712105003. [26] X. H. Song, D. X. Niu, C. Q. Ye, y D. L. Lu, «Survey on the Quality Management System of Power Distribution Projects Based on the Theory of TQM», Appl. Mech. Mater., vol. 108, pp. 3034, oct. 2011, doi: 10.4028/www.scientific.net/AMM.108.30. 
[27] J. A. Salvador-Oliván y C. Agustín-Lacruz, «Correlación entre indicadores bibliométricos en revistas de Web of Science y Scopus», Rev. Gen. Inf. Doc., vol. 25, n.o 2, pp. 341-359, dic. 2015, doi: 10.5209/rev_RGID.2015.v25.n2.51241.

[28] G. Khiste y R. Paithankar, «Mapping of the Literature on "Bibliometric" By using Science Direct during 2005-2016», New Man Int. J. Multidiscip. Stud., vol. 4, pp. 89-93, sep. 2017.

[29] M. Cubillos Rodríguez y D. R. Rodríguez, «El concepto de calidad: Historia, evolución e importancia para la competitividad», Rev. Univ. Salle, vol. 2009, n.o 48, pp. 80-99, ene. 2009.

[30] F. Camps Pons, «Estado de conocimiento sobre la integración de sistemas de gestión a partir del análisis bibliométrico de artículos publicados en revistas JCR», oct. 2016, Accedido: may 18, 2021. [En línea]. Disponible en: https://upcommons.upc.edu/handle/2117/99333

[31] P. Jordán y R. Miguel, «La relación entre el sector industrial y el tamaño de empresa con las prácticas de la calidad total y el desempeño organizacional», Pontif. Univ. Católica Perú, sep. 2011, Accedido: may 18, 2021. [En línea]. Disponible en: http://tesis.pucp.edu.pe/repositorio/handle/20.500.12404/782 